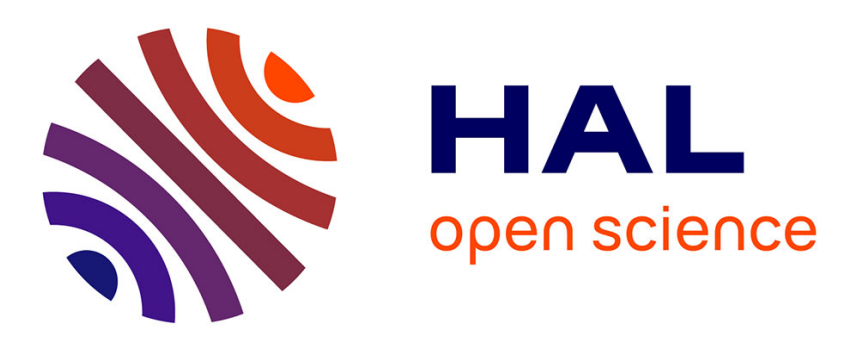

\title{
Relationship between burgers circuit, volterra process and homotopy groups
}

\author{
M. Kléman
}

\section{To cite this version:}

M. Kléman. Relationship between burgers circuit, volterra process and homotopy groups. Journal de Physique Lettres, 1977, 38 (10), pp.199-202. 10.1051/jphyslet:019770038010019900 . jpa-00231359

\section{HAL Id: jpa-00231359 https://hal.science/jpa-00231359}

Submitted on 1 Jan 1977

HAL is a multi-disciplinary open access archive for the deposit and dissemination of scientific research documents, whether they are published or not. The documents may come from teaching and research institutions in France or abroad, or from public or private research centers.
L'archive ouverte pluridisciplinaire HAL, est destinée au dépôt et à la diffusion de documents scientifiques de niveau recherche, publiés ou non, émanant des établissements d'enseignement et de recherche français ou étrangers, des laboratoires publics ou privés. 
Classification

Physics Abstracts

$1.360-7.160-7.489$

\title{
RELATIONSHIP BETWEEN BURGERS CIRCUIT, VOLTERRA PROCESS AND HOMOTOPY GROUPS
}

\author{
M. KLÉMAN \\ Laboratoire de Physique des Solides, Bâtiment 510, 91405 Orsay, France
}

(Reçu le 28 décembre 1976, accepté le 21 avril 1977)

\begin{abstract}
Résumé. - On compare les deux classifications connues des singularités linéaires dans les milieux ordonnés, à savoir par les éléments de groupe de symétrie du milieu ou par les classes d'équivalence du groupe fondamental de la variété des états internes. Ceci conduit à une nouvelle définition du circuit de Burgers, qui généralise celle bien connue pour les dislocations de translation, et à une analyse nouvelle de la notion de processus de Volterra sur la surface de coupure.

Abstract. - A comparison is made of the different classifications of linear singularities in ordered media, viz. by use of the elements of the symmetry group of the medium or by the equivalence classes of the fundamental group of the manifold of internal states. It allows for a new definition of the Burgers circuit, which generalizes that one known for translation dislocations and for a new analysis of the notion of Volterra process on the cut surface.
\end{abstract}

There are two classifications of linear defects (dislocations) in ordered media :

- By the homotopy group $\Pi_{1}(\mathrm{~V})$ of the manifold of internal states [1]. $\mathrm{V}$ is the set of the cosets of the quotient $\mathbf{G} / \mathrm{H}$ [2], where $\mathrm{H}$ is the symmetry group of the medium and $G$ a group whose action leaves the medium invariant as a whole. $G$ is generally a $\mathrm{Lie}$ group, such as the Euclidean group E(3), or the group of rotations $O(3)$ of a solid body. Physically, G is the group of symmetry of the high temperature phase, and acts transitively on V.

- By the Volterra process [3] : to each element $\mathrm{h} \in \mathrm{H}$ corresponds an operation in the perfect medium on a cut surface $\Sigma$, bounded by a line L, whose result is a dislocation line. Reciprocally, it is assumed that an element $\mathrm{h} \in \mathrm{H}$ can be associated with any dislocation $\mathrm{L}$ present in the ordered medium. This assumption will be the object of the discussions below, where it will be shown that the operation on the cut surface $\Sigma$ itself has to be qualified.

The classification by the equivalence classes in the group $\Pi_{1}(\mathrm{~V})$ leads to the notion of topologically stable defects. The physical stability is another problem, whose discussion needs the calculation of dislocation energies for various configurations (shape stability, core stability, etc... for examples in a nematic, see [4]). The boundary conditions on the cut surface $\Sigma$ play a crucial role in these calculations. The nature of these boundary conditions is well known in the case of translation dislocations, where we just have to write that the difference in the displacements $\mathbf{u}_{2}-\mathbf{u}_{1}$ of the two lips of $\Sigma$ is equal to a translation vector of the symmetry group :

$$
\mathbf{u}_{2}-\mathbf{u}_{1}=\mathbf{b}
$$

but it will be shown here that the situation is more complex in the general case.

In the course of this letter, we shall first discuss a generalization of the notion of Burgers path which appears necessary when the line singularity involves a rotational symmetry element; then we shall show that to each symmetry element (to which is generally attached a given Volterra process) correspond several non-equivalent Burgers path whose relevant homotopy classes form one (or several) conjugacy classes of $\Pi_{1}(\mathrm{~V})$. The physical meaning of these conjugacy classes as well as that of commutators of $\Pi_{1}(\mathrm{~V})$ will be discussed in some examples of singularity lines which are linked or not linked. Finally we shall show that the consideration in the distorted medium of a surface $\Sigma$, bounded by the line, and locus of the origins of a set of Burgers circuits, enables us to distinguish between lines which can be described as due to a Volterra process (for which boundary conditions of the type of eq. (1) can be written; the surface in that case is the image in the distorted medium of the Volterra cut surface), and those which cannot (in that case some topological properties of $\Sigma$ defined below should be added to the boundary conditions). 
Burgers circuit and Burgers path (definition). Consider a singularity line 1 in the distorted medium, and a loop $\gamma$ circling 1. $\gamma$ is the Burgers circuit, 1 and $\gamma$ will be assumed oriented according to the right hand screw rule. Let $\mathbf{M}$ be an origin on $\gamma$; consider a neighbouring point $\mathbf{M}+\mathrm{dM}$ on $\gamma$. Call $\mathrm{dg} \in \mathrm{G}$ the operation which enables us to pass from the local orientation $\mu$ in $M$ to the local orientation $\mu+\mathrm{d} \mu$ in $\mathrm{M}+\mathrm{dM} . \mu$ is a point in $\mathrm{V}\left({ }^{1}\right)$, and we have

$$
\mu+\mathrm{d} \mu=\mathrm{dg} \cdot \mu
$$

$\mathrm{dg}$ is a point in $\mathrm{G}$, near the identity element 0 . When completing the circuit $\gamma$, going from $\mathbf{M}$ to $\mathbf{M}$, the integral path of $\mathrm{dg}$ is a path $\Gamma$ going from 0 to $\mathrm{h} \in \mathrm{G}$, where $h$ is clearly an element in the symmetry group $\mathrm{H}(\mathrm{M})$ of the medium.

$\Gamma$ is in general an open path. We call it the Burgers path : it bears some analogy with the image in the perfect crystal of the well-known Burgers circuit of translation dislocations : in that case $\mathbf{G}$ is the Euclidean space $R^{3}$ of all possible translations, $H$ is a subgroup of $G$ consisting of a periodic lattice (the Bravais lattice), $G / H=T^{3}$ (the torus with dimension 3), and $h$ the Burgers vector. Hence the Burgers path of a translation dislocation is an image of the Burgers circuit in the Bravais lattice (which is not different from the perfect crystal as far as we are concerned).

Note that the group of symmetry in $\mathbf{M}+\mathrm{dM}$ is :

$$
H(M+d M)=d g \cdot H(M) \cdot d g^{-1} \text {. }
$$

After completion of $\gamma$, one has :

$$
\mathrm{H}(\mathrm{M}) \rightarrow \mathrm{h} \cdot \mathrm{H}(\mathrm{M}) \cdot \mathrm{h}^{-1} \text {. }
$$

This is a mapping of $\mathrm{H}(\mathrm{M})$ onto itself, but not an identity mapping, unless $\mathrm{H}(\mathrm{M})$ is Abelian. The only element which in all cases maps identically is $h$ : the ordered medium has been rotated by an element of symmetry $\mathrm{h}$ and the presence of the line 1 lifts the $\mathrm{h}$ degeneracy of the crystal.

Relationship between Burgers path and the elements of $\Pi_{1}(\mathrm{~V})$. - We wish to study the set of all paths $\Gamma(\mathrm{h})$ in $\mathrm{G}$ which start at 0 and finish at $\mathrm{h}$. All these paths project into closed paths in $\mathrm{V}=\mathrm{G} / \mathrm{H}$. We shall loosely call homotopy class of a path $\Gamma(\mathrm{h})$ the homotopy class $\alpha(h)$ of its projection in $V$, and show that the set of all $\alpha(\mathrm{h})$ (for a given $\mathrm{h}$ ) is in 1-1 correspondence with the so-called derived group of $\Pi_{1}(\mathrm{~V})$.

If $\mathrm{G}$ is a Lie group, $\Pi_{1}(\mathrm{G})$ is Abelian; we represent the $n$ (say) elements of $\Pi_{1}(G)$ by $n$ closed loops in $G$ all passing through the identity element 0 . To each loop there corresponds a homotopy class $\alpha_{i}(0)$

( $\left.{ }^{1}\right)$ Any point $\mu$ in $V$ defines an absolute orientation of the ordered medium. Two points $\mu$ and $\mu^{\prime}$ are related by one (or many) operations $\mathrm{g} \in \mathrm{G}$, such that $\mu^{\prime}=\mathrm{g} \mu$, $\mathrm{g}$ is the relative orientation of $\mu^{\prime}$ with respect to $\mu$. Hence $G$ is also the manifold of relative orientations.
( $i=1,2, \ldots, n$ ) belonging to $\Pi_{1}(\mathrm{~V})$. It is clear that the set $\mathrm{D}=\bigcup_{i} \alpha_{i}(0)$ is an invariant subgroup of $\Pi_{1}(\mathrm{~V})$, since the products $\alpha_{i}(0) \alpha_{j}(0)$ and $\alpha_{k}^{-1}(\mathrm{~g})$ $\alpha_{i}(0) \alpha_{k}(\mathrm{~g})$ are closed loops in $G$, and since $\alpha_{i}^{-1}(0)$ and the trivial loop (homotopic to zero in $\mathrm{V}$ ) are in this set.

Let us notice that $\alpha(\mathrm{g})$ is also the homotopy class of any path denoted $\Gamma_{\mathrm{h}}(\mathrm{g})$, beginning at a given element $\mathrm{h} \in \mathrm{G}$ and such that any point of $\Gamma_{\mathrm{h}}(\mathrm{g})$ is obtained by the left translation h.k of its counter image $k$ in $\Gamma(\mathrm{g})$, the homotopy class of $\Gamma(\mathrm{g})$ being $\alpha(\mathrm{g})$. We write symbolically :

$$
\Gamma_{\mathrm{h}}(\mathrm{g})=\mathrm{h} \Gamma(\mathrm{g}) .
$$

Hence the homotopy class $\alpha_{i}(\mathrm{~g}) \alpha_{j}(\mathrm{~h})$ can be obtained as the homotopy class of a path

$$
\left[\mathrm{h} \Gamma_{i}(\mathrm{~g})\right] \cdot \Gamma_{j}(\mathrm{~h}) .
$$

Consider now the closed loop

$$
\left[\mathrm{g} \Gamma_{i}\left(\mathrm{~g}^{-1}\right)\right]\left[\mathrm{hg} \Gamma_{j}\left(\mathrm{~h}^{-1}\right)\right] \cdot\left[\mathrm{h} \Gamma_{i}(\mathrm{~g})\right] \cdot \Gamma_{j}(\mathrm{~h}) .
$$

Its class of homotopy is

$$
\alpha_{i}^{-1}(\mathrm{~g}) \alpha_{j}^{-1}(\mathrm{~h}) \alpha_{i}(\mathrm{~g}) \alpha_{j}(\mathrm{~h})
$$

which is a commutator of $\Pi_{1}(V)$ and is in $D$, by construction. $\mathrm{D}$ is therefore the derived group of $\Pi_{1}(\mathrm{~V})$ or, eventually, an invariant subgroup of $\Pi_{1}(\mathrm{~V})$ which contains the derived group.

We can partition $\Pi_{1}(\mathrm{~V})$ in cosets $\alpha(\mathrm{h}) . \mathrm{D}$, which can be written

$$
\Pi_{1}(\mathrm{~V})=\mathrm{D}+\alpha(\mathrm{g}) \cdot \mathrm{D}+\alpha(\mathrm{h}) \cdot \mathrm{D}+\cdots
$$

where $g, h, \ldots$ are the different elements of the symmetry group $\mathrm{H}$, and $\alpha(\mathrm{g})$ is any homotopy class of the set of paths $(\Gamma(\mathrm{h}))$. Each coset $\alpha(\mathrm{h}) \mathrm{D}$ contains $n$ elements, all corresponding to the same Volterra element $\mathrm{h}$. Notice finally that $\Pi_{1}(\mathrm{~V}) / \mathrm{D}$ is an Abelian group :

$$
\Pi_{1}(\mathrm{~V}) / \mathrm{D}=\mathrm{H}_{1}(\mathrm{~V}, \mathrm{Z})
$$

(which is the first homology group of $\mathrm{V}$, if $\mathrm{D}$ is the derived group), and that each coset contains whole conjugacy classes of elements of $\Pi_{1}(\mathrm{~V})$. This means that, if $\alpha(\mathrm{h})$ is a constant element in $\Pi_{1}(\mathrm{~V})$, and $\alpha(\mathrm{g})$ a variable one, the element $\alpha(\mathrm{g}) \alpha(\mathrm{h}) \alpha^{-1}(\mathrm{~g})$ is in the same coset of $\Pi_{1}(\mathrm{~V}) / \mathrm{D}$ as $\alpha(\mathrm{h})$, and is therefore the homotopy class of a particular path of the set $\Gamma(\mathrm{h})$.

Physical meaning of commutators and conjugacy classes in $\Pi_{1}(\mathrm{~V})$. - i) Borromean knot (Fig. 1). Consider two defect loops $l_{1}$ and $l_{2}$ linked by a circuit which circles both of them twice but in opposite directions. The homotopy class in $\mathrm{V}$ of this circuit is

$$
\alpha(B)=a_{1}^{-1} a_{2}^{-1} a_{1} a_{2}
$$

which clearly depends on the origin on (B), and on the orientation of $(B)$. Change origin and orientation 


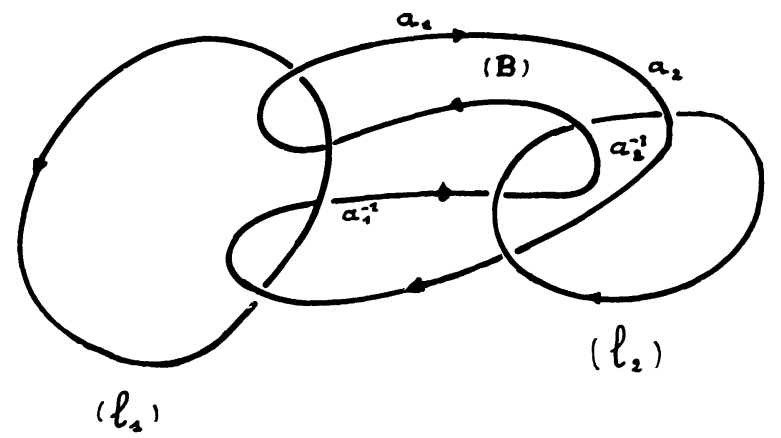

Fig. 1. - Borromean knot linking two singularity lines $l_{1}$ and $\mathbf{l}_{2}$. The homotopy class of (B) is a commutator of the fundamental group $\Pi_{1}(\mathrm{~V})$.

on B : $\alpha(\mathrm{B})$ belongs to a subset of one commutator class $\mathrm{C}$ (which is a class of conjugacy), or possibly two commutator classes (if $\mathrm{C}$ is not ambivalent) $\left(^{2}\right.$ ).

ii) Topological interaction between loops : multiply both sides of eq. (9) by $a_{1}$ :

$$
a_{1} \alpha(B)=a_{2}^{-1} a_{1} a_{2} \text {. }
$$

This quantity is the homotopy class of a loop circling $1_{1}$ once and $l_{2}$ twice but in opposite directions. It is therefore an homotopy class of $1_{1}$. The conjugacy class of $\mathrm{a}_{1}$ represents therefore the possible topological interactions with other loops present in the medium.
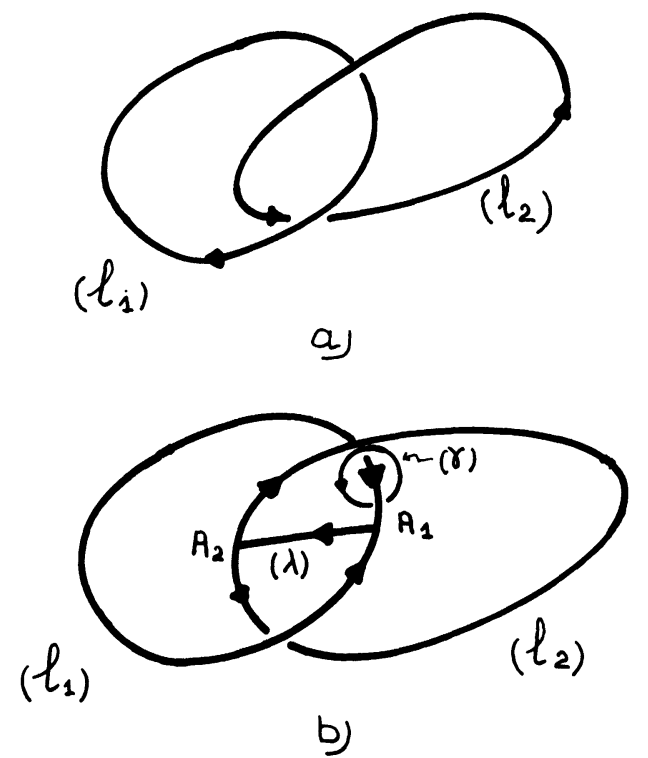

FIG. 2. - The homotopy classes of two linked lines must commute (a). If they do not, the two lines must be joined by a line whose homotopy class is a commutator $(b)$.

iii) Linked lines : consider two linked lines $l_{1}$ and $1_{2}$ (Fig. 2a). It is not difficult to show that the homotopy classes $a_{1}$ and $a_{2}$ of $l_{1}$ and $l_{2}$ obey the equation

$$
a_{1}=a_{2} a_{1} a_{2}^{-1}
$$

(2) A conjugacy class $\mathrm{C}$ is said to be ambivalent if it contains the inverses of all its elements. so that $a_{1}$ and $a_{2}$ must commute. If they do not, the two dislocations $l_{1}$ and $l_{2}$ must be joined by a segment of dislocation line $(\lambda)$, whose homotopy class clearly must be a commutator of the homotopy classes of $l_{1}$ and $l_{2}$

$$
\alpha=a_{1} a_{2} a_{1}^{-1} a_{2}^{-1} .
$$

This expression has to be understood as the product of the homotopy class $a_{1}$ by the homotopy class $a_{2} a_{1}^{-1} a_{2}^{-1}$, i.e. the homotopy class of the line $l_{1}$ oriented in the opposite direction, and after the closed circuit $\gamma_{1}$ has been rotated all along $l_{1}$, from one side of the node $A_{1}$ to the opposite side (see Fig. 2b). The product

$$
\alpha^{\prime}=\left(a_{2} a_{1}^{-1} a_{2}^{-1}\right) a_{1}
$$

is in the same homotopy class as $\alpha$; hence the order in the product of the homotopy classes building $(\lambda)$ does not matter. But if we consider now the node $A_{2}$, one gets, for example

$$
\alpha^{\prime \prime}=a_{1} a_{2}^{-1} a_{1}^{-1} a_{2}
$$

which is not in the same commutator class as $\alpha$ and $\alpha^{\prime}$, but in the commutator class containing the inverse $\alpha^{-1}$. Hence, if we want $(\lambda)$ to be uniquely defined, this requires that $(\lambda)$ be a non-orientable line, and that its commutator class be ambivalent. Examples can easily be worked out (the - I element in the homotopy group of biaxial nematics [2]).

iv) Relative displacement of two lines : Displace a line $l_{1}$ about a line $l_{2}$, on a closed curve homotopic to a closed circuit surrounding $1_{2}$ (this can easily be visualized : take as an example two parallel straight lines and completely rotate one about the other). The homotopy class $a_{1}$ changes to $a_{2} a_{1} a_{2}^{-1}$ (for mathematical justifications, see ref. [6], p. 84)

$$
a_{1} \rightarrow a_{1}^{\prime}=a_{2} a_{1} a_{2}^{-1} \text {. }
$$

An example due to Frank (see ref. [5]) shows a case in which $a_{1}^{\prime}=a_{1}^{-1}$ (the line has changed sign, due to the fact that the conjugacy class of $a_{1}$ is ambivalent).

The role of the cut surface in the boundary conditions. - Consider a line 1 in a distorted medium, and a surface $\Sigma$ bounded by $1 . \Sigma$ will be for us the locus of the origin $\mathbf{M}$ of the closed circuits $\gamma$ on which the homotopy class of 1 is computed (see Fig. 3). To the whole (continuous) set $\gamma$ corresponds a continuous set of Burgers circuits $\Gamma$ on $\mathrm{G}$, whose extremities $\mathrm{h}$ describe a two-dimensional manifold $\sigma$.

To define the topological properties of $\sigma$ is important in the definition of 1 . It can be shown for example that, in the case of figure $4 a, \sigma$ is homotopic to a point, whereas in the case of figure $4 b, \sigma$ is homotopic to a loop which cannot be continuously shrunk to a point.

But, more generally, let us assume that $h_{0}$ is the element in $\mathrm{H}$ at the extremity of a particular Burgers circuit. All other $h$ are in the conjugacy class of $h_{0}$ 


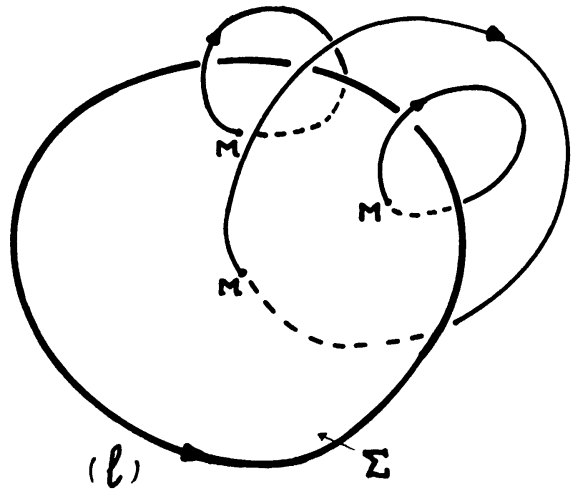

FIG. 3. - Cut surface and closed loops beginning on the cut surface.

in $\mathrm{G}$, viz. $\mathrm{gh}_{0} \mathrm{~g}^{-1}$. Call $\mathrm{N}$ the manifold which is defined by this conjugacy class. We are interested in the homotopy class of $\sigma$ in $\mathrm{N}$ ( $\sigma$ is of course in $\mathrm{N}$ ).

- If $\sigma$ is a closed manifold, the relevant quantity is $\Pi_{2}(\mathrm{~N})$. For example, taking the case of nematics, where $\mathrm{h}$ is in $\mathrm{SO}(3)$ a rotation of $\pi, \mathrm{N}=\mathrm{P}^{2}$ and $\Pi_{2}\left(\mathrm{P}^{2}\right)=\mathrm{Z}$. There is therefore an infinity of closed $\sigma$, hence an infinity of types of topologically different boundary conditions on $\Sigma$. We do not know as yet of any physical situation in which such a defect has been observed.

- If $\sigma$ is not closed, there are several possibilities, each of them corresponding to an element of the group $\Pi_{1}(\mathrm{~N})$. In a nematic phase $\Pi_{1}\left(\mathrm{P}_{2}\right)=\mathrm{Z}_{2}$, so that there are two possibilities :

i) if $\sigma$ is homotopic to a point, it is possible to achieve the line by using one element of symmetry only on the cut surface : this is the classical Volterra process. The process proposed by de Gennes for

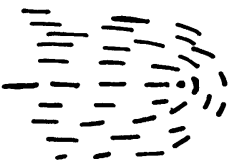

a)
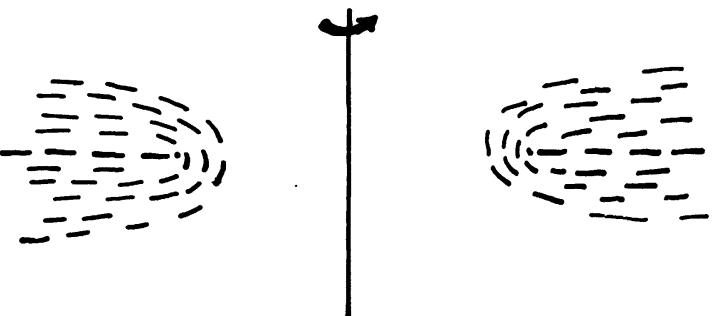

b)

Fig. 4. - Two types of lines in nematics (note that $: a$ ) is a straight line, b) a circular line), corresponding to two non-homotopic mappings of $\Sigma$ on $\mathrm{N} \in \mathrm{G}$.

nematics [7] enters this case. Figure $4 a$ is a wellknown example (wedge line). $\Sigma$ is therefore a true image of possible cut surface in the perfect medium, drawn before completion of the Volterra process;

ii) if $\sigma$ is homotopic to a line, the Volterra process is irrelevant. Figure $4 b$ represents a line drawn in a nematic phase, which cannot be explained by the Volterra process (viz. one symmetry element) but is defined by non-trivial topological properties of the image of $\Sigma$ in G.

I thank Prof. F. C. Frank for stimulating discussions.

\section{References}

[1] Toulouse, G. and Kléman, M., J. Physique Lett. 37 (1976) L-149;

Rogula, D., in Trends in Applications of Pure Mathematics to Mechanics, edited by G. Fichera (Pitman Publishing) 1976.

[2] Kléman, M., Michel, L. and Toulouse, G., J. Physique Lett. 38 (1977) L-195.

[3] Friedel, J., Dislocations (Pergamon Press) 1964.

[4] Kleman, M., Adv. Liq. Cryst. 1 (1975) 267.
[5] Friedel, J., Kléman, M., Nat. Bur. Stand. (U.S.), Spec. Publ. 317 (1969) 607.

[6] STEENROD, N., The topology of fiber bundles (Princeton U.P.) 1957 .

[7] Friedel, J. and De Gennes, P. G., C. R. Hebd. Séan. Acad. Sci. 268 (1969) 257.

[8] Kléman, M., Points, lignes, parois dans les fluides anisotropes et les solides cristallins, monography (J. Physique ed.) 1977. 